\title{
The geometric method for solving a heat equation
}

\author{
Oleg Stelia ${ }^{\mathrm{a}}$, Igor Stelia ${ }^{\mathrm{b}}$
}

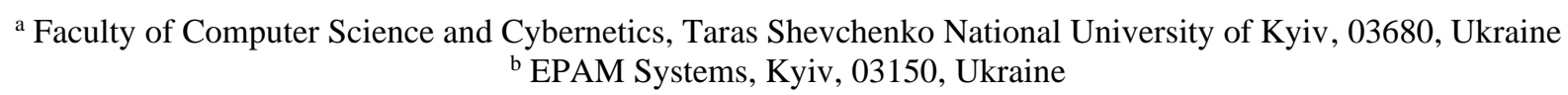

E-mail: oleg.stelya@gmail.com, igor.stelia@gmail.com

\begin{abstract}
In the proposed publication, we present a geometric method for solving a boundary value problem for a nonstationary heat equation. The equivalence of the geometric solution to an explicit difference method is shown with the corresponding initial parameters. There are considered the first and second boundary value problems. The physical interpretation of the method is based on local balance.
\end{abstract}

MSC: $65 \mathrm{~N} 99$

Keywords: Explicit difference scheme, Boundary value problem, Geometrical representation, Physical interpretation

\section{Introduction}

Let us consider initial-boundary value problem for the heat equation

$$
\begin{aligned}
& \frac{\partial u(x, t)}{\partial t}=D \frac{\partial^{2} u(x, t)}{\partial x^{2}}+f(x), \quad x \in(a, b), t>0, \\
& u(x, t)=U(x), \quad x=a, \quad t>0, \\
& u(x, t)=U(x), \quad x=b, \quad t>0, \\
& u(x, 0)=U(x), \quad x \in[a, b] .
\end{aligned}
$$

An explicit difference scheme $(f(x)=0)$ for equation (1) [1] is written as follows:

$$
\frac{u_{i}^{k+1}-u_{i}^{k}}{\Delta t}=D \frac{u_{i-1}^{k}-2 u_{i}^{k}+u_{i+1}^{k}}{h^{2}},
$$

where $u_{i}^{k}=u\left(x_{i}, t_{k}\right)$ is a grid function,

$$
\begin{aligned}
& a=x_{1}<x_{2}<\ldots<x_{i}<\ldots<x_{N}=b, \\
& h=x_{i+1}-x_{i}=\text { const }, i=\overline{1, N-1}, \Delta t=t_{k+1}-t_{k}=\text { const }, k=0,1,2, \ldots .
\end{aligned}
$$


Scheme (5) is conditionally stable with respect to perturbation of the initial data. The stability condition is written as $\Delta t \leq \frac{h^{2}}{2 D}[1]$.

The difference solution of the problem (1) - (4) is calculated as follows:

$u_{1}^{k+1}=U\left(x_{1}\right)$,

$u_{i}^{k+1}=u_{i}^{k}+\frac{D \Delta t}{h^{2}}\left(u_{i-1}^{k}-2 u_{i}^{k}+u_{i+1}^{k}\right), \quad i=\overline{2, N-1}$,

$u_{N}^{k+1}=U\left(x_{N}\right)$,

где $u_{i}^{0}=U\left(x_{i}\right), i=\overline{1, N}$.

Let us assume that conditions of the first kind are given for equation (1) at the boundary points:

$\frac{\partial u(a, t)}{\partial x}=0, \quad \frac{\partial u(b, t)}{\partial x}=0$.

Difference conditions for (7) can be written as follows [2]:

$\frac{u_{1}^{k+1}-u_{1}^{k}}{\Delta t}=\frac{2}{h}\left(u_{2}^{k}-u_{1}^{k}\right) / h$,

$\frac{u_{N}^{k+1}-u_{N}^{k}}{\Delta t}=-\frac{2}{h}\left(u_{N}^{k}-u_{N-1}^{k}\right) / h$.

\section{Geometric method for solving the equation (1)}

First boundary value problem. Let the initial condition of the equation (1) is given (Figure 1, red line). The node values $u_{i}^{0}, i=\overline{1, N}$ are marked with red dots. The calculation of the discrete solution at the points $\left(x_{i}, t_{1}\right), i=\overline{2, N-1}$ is carried out in two steps:

Step 1 consists in finding the midpoints of the segments (Figure 1, red intervals) which connect the points $u_{i}^{0}, i=\overline{1, N}$. The values at these points are denoted $y_{i-1 / 2}$ where

$y_{i-1 / 2}=\left(u_{i-1}^{0}+u_{i}^{0}\right) / 2, \quad i=\overline{2, N}$.

Step 2 consists in connecting the points $y_{i-1 / 2}, i=\overline{2, N}$ (Figure 1, blue dotted line) and calculating values $u_{i}^{1}$ in points $x_{i}, i=\overline{2, N-1}$

$$
u_{i}^{1}=\left(\left(u_{i-1}^{0}+u_{i}^{0}\right) / 2+\left(u_{i}^{0}+u_{i+1}^{0}\right) / 2\right) / 2=\left(u_{i-1}^{0}+2 u_{i}^{0}+u_{i+1}^{0}\right) / 4=u_{i}^{0}+\left(u_{i-1}^{0}-2 u_{i}^{0}+u_{i+1}^{0}\right) / 4 .
$$

The values at the boundary points remain unchanged.

Expression (10) can be rewritten as: 
$\frac{u_{i}^{1}-u_{i}^{0}}{1 / 4}=u_{i-1}^{0}-2 u_{i}^{0}+u_{i+1}^{0}, \quad i=\overline{2, N-1}$.

For $D=h^{2}, \Delta t=1 / 4, k=0$ formula (6) coincides with (11). The solution of the boundary value problem (1) - (4) calculated at the moment of time $t_{k}, k=1$ by the graphical method coincides with the one calculated according to the scheme (6). In this case, the stability condition is satisfied.

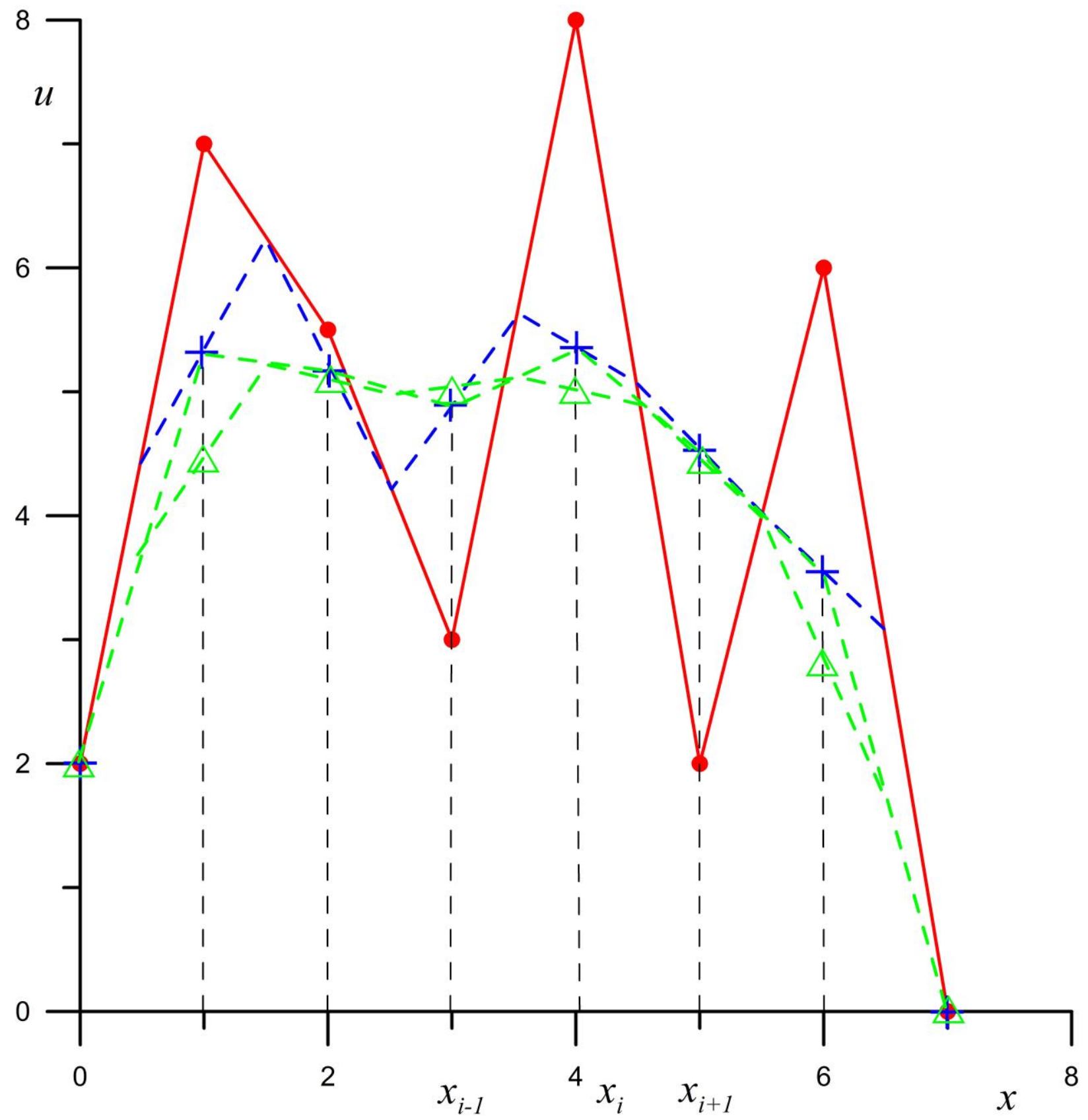

Fig. 1. Graphical method for solving equation (1) $(x \in[0,7], N=8, h=1)$. First boundary value problem. $\mathbf{R}$ - initial condition $U(x), x \in[0,7]$; $\mathbf{-}$ solution computed at time $t_{k}, k=1$; - solution computed at time $t_{k}, k=2$. 
The green line in Figure 1 shows a schematic representation of the graphical calculation of the solution at the time $t_{k}, k=2$. The solution at the moments of time $t_{k}, k=3,4 \ldots$ can be calculated analogously.

Second boundary value problem. Physical interpretation of the solution. Let conditions (7) are given at the points $x=a, x=b$.

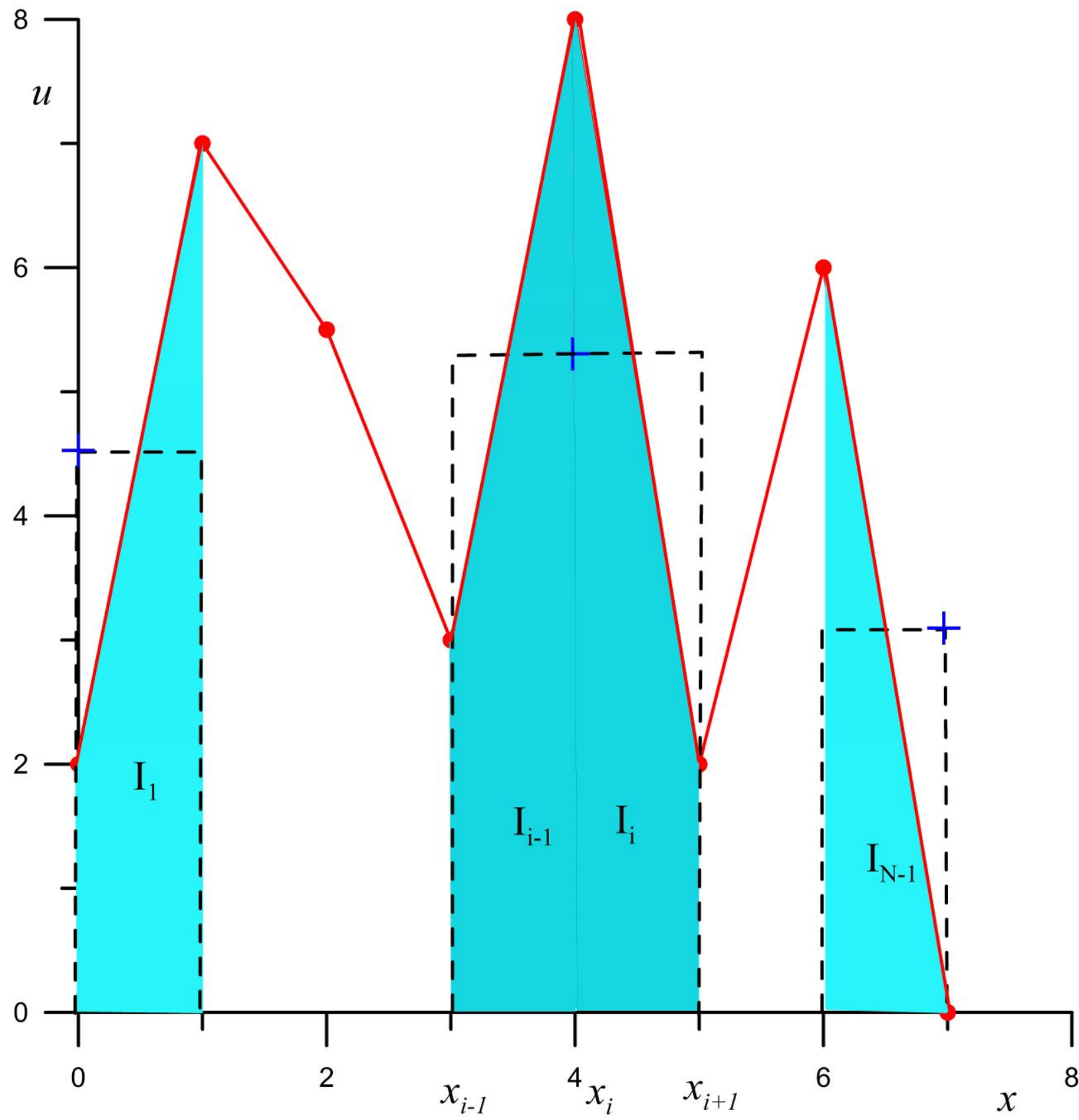

Fig. 2. Physical interpretation of the graphics solution. Second boundary value problem.

In Figure 2, the initial condition $U(x)$ with nodal values $u_{i}^{0}, i=\overline{1, N}$ is marked in red. The sum of the areas of the figures $I_{i}, i=\overline{1, N-1}$ (marked in blue) equals to the 
integral $\int_{a}^{b} U(x) d x$. The solution at the moment of time $t_{1}$ in points $x_{i}, i=\overline{2, N-1}$ is calculated as the height of a rectangle equal in area to the sum of the areas of the figures $I_{i-1}$ and $I_{i}$ :

$$
\begin{aligned}
& u_{i}^{1}=I_{i-1}+I_{i}=\left(\left(u_{i-1}^{0}+u_{i}^{0}\right) h / 2+\left(u_{i}^{0}+u_{i+1}^{0}\right) h / 2\right) / 2 h= \\
& =\left(u_{i-1}^{0}+2 u_{i}^{0}+u_{i+1}^{0}\right) / 4=u_{i}^{0}+\left(u_{i-1}^{0}-2 u_{i}^{0}+u_{i+1}^{0}\right) / 4 .
\end{aligned}
$$

Expressions (10) and (12) coincide.

Let's calculate the solution at one of the boundary points, for example, at a point $x_{N}$.

The area of the figure $I_{N-1}$ (Figure 2) is calculated as follows:

$$
I_{N-1}=\left(u_{N}^{0}+u_{N-1}^{0}\right) h / 2 .
$$

The solution at the point $\left(x_{N}, t_{1}\right)$ is calculated as a height of a rectangle equal in area to the figure $I_{N-1}$ :

$$
\begin{aligned}
& u_{N}^{1}=\left(u_{N}^{0}+u_{N-1}^{0}\right) h / 2 h=\left( \pm 2 u_{N}^{0}+u_{N}^{0}+u_{N-1}^{0}\right) / 2=u_{N}^{0}+\left(-2 u_{N}^{0}+u_{N}^{0}+u_{N-1}^{0}\right) / 2= \\
& =u_{N}^{0}-\left(u_{N}^{0}-u_{N-1}^{0}\right) / 2 .
\end{aligned}
$$

Hence, we get the condition:

$$
\frac{u_{N}^{1}-u_{N}^{0}}{1 / 4}=-2\left(u_{N}^{0}-u_{N-1}^{0}\right) \text {. }
$$

The obtained condition coincides with the difference condition (9) at $k=0$.

The solution at a point $\left(x_{1}, t_{1}\right)$ is calculated analogously. It is easy to obtain a solution for $f(x) \neq 0$.

Physical interpretation. Physical interpretation is based on heat conservation for the figures $I_{i}, i=\overline{1, N-1}$ (Figure 2). It is assumed that for figures $I_{1}, I_{N-1}$ and for sums of figures $I_{i-1}+I_{i}, i=\overline{2, N-1}$, there is no heat exchange with neighboring figures. Let the temperature in the selected figure at the initial moment of time $t_{0}$ be unevenly distributed. Let us suppose that the temperature is setting and becoming constant. After that, the resulting constant is assigned as a solution at the nodal point $x_{i}$ at a time $t_{1}$. The procedure repeats for the next points in time.

\section{Conclusion}

The constructed geometric method for solving the first and second boundary value problems for the heat equation gives a good illustration of the use of an explicit finite difference scheme. The physical interpretation corresponds with the process of heat conduction or diffusion. The proposed research has a methodological interest for the 
study and illustration of difference methods for solving boundary value problems. The method can be used for computer visualization.

\section{References}

1. Robert D. Richtmyer, K. W. Morton, Difference Methods for Initial-Value Problems 2nd Edition, by Krieger Pub Co, 1994, 420 p.

2. Samarskii A.A. The Theory Of Difference Schemes, New York: Marcel Dekker, Inc. , 2001, 762 p. https://doi.org/10.1201/9780203908518. 